\title{
Polarimetric imaging of NGC 1068 at high angular resolution in the near infrared
}

\author{
Direct evidence of an extended nuclear torus ${ }^{\star, \star \star}$
}

\begin{abstract}
D. Gratadour, D. Rouan, L. Grosset, A. Boccaletti, and Y. Clénet
LESIA, Observatoire de Paris, CNRS, UPMC and Univ. Paris Diderot, France

e-mail: damien.gratadour@obspm.fr

Received 18 May 2015 / Accepted 29 June 2015

ABSTRACT

Aims. One of the main observational challenges for investigating the central regions of active galactic nuclei (AGN) at short wavelengths, using high angular resolution, and high contrast observations, is to directly detect the circumnuclear optically thick material hiding the central core emission when viewed edge-on. The lack of direct evidence is limiting our understanding of AGN, and several scenarios have been proposed to cope for the diverse observed aspects of activity in a unified approach.

Methods. Observations in the near-infrared spectral range have shown themselves to be powerful for providing essential hints to the characterisation of the unified model ingredients because of the reduced optical depth of the obscuring material. Moreover, it is possible to trace this material through light scattered from the central engine's closest environment, so that polarimetric observations are the ideal tool for distinguishing it from purely thermal and stellar emissions.

Results. Here we show strong evidence that there is an extended nuclear torus at the center of NGC 1068 thanks to new adaptiveoptics-assisted polarimetric observations in the near-infrared. The orientation of the polarization vectors proves that there is a structured hourglass-shaped bicone and a compact elongated $(20 \times 60 \mathrm{pc})$ nuclear structure perpendicular to the bicone axis. The linearly polarized emission in the bicone is dominated by a centro-symmetric pattern, but the central compact region shows a clear deviation from the latter with linear polarization aligned perpendicular to the bicone axis.
\end{abstract}

Key words. galaxies: active - galaxies: Seyfert - techniques: polarimetric - techniques: high angular resolution

\section{Introduction}

The unified model of active galactic nuclei, which has been largely accepted, explains the extreme energy production in a compact region by the presence of a supermassive black hole (a few million to billions of solar masses), which is continuously fueled through an accretion disk and which strongly irradiates its close environment mainly at short wavelengths. To cope with the diverse observed aspects of activity, a key ingredient is the presence of circumnuclear, optically thick material, arranged in an anisotropic manner and hiding the central core emission when viewed edge-on (Antonucci 1993). As one of the closest active galaxies (15 Mpc), NGC 1068 is the ideal laboratory for studying nuclear activity. While indirect evidence has been found in this object, thereby corroborating the unified model (Antonucci \& Miller 1985; Raban et al. 2009), many unknowns remain as to the nature and distribution of the obscuring material (Nenkova et al. 2002). Recently, the complexity of the circumnuclear environment has been revealed by high angular resolution broad

\footnotetext{
* Figure 2 is available in electronic form at http://www . aanda.org

$\star \star$ Data obtained with the SPHERE an instrument designed and built by a consortium consisting of IPAG (France), MPIA (Germany), LAM (France), LESIA (France), Laboratoire Lagrange (France), INAF Osservatorio di Padova (Italy), Observatoire de Genève (Switzerland), ETH Zurich (Switzerland), NOVA (Netherlands), ONERA (France), and ASTRON (Netherlands) in collaboration with ESO.
}

band observations in the near-IR (Rouan et al. 2004; Gratadour et al. 2006; Exposito et al. 2011). In this Letter, we present the new elements introduced by polarimetry.

\section{Observations and data processing}

The core of NGC 1068 was observed with the SPHERE instrument on the Very Large Telescope, under the science verification program, using the infrared camera IRDIS (Langlois et al. 2014) in its polarimetric mode. SPHERE has been designed for hunting exoplanets through direct imaging and is equipped with the extreme adaptive optics system SAXO (Beuzit et al. 2008), providing diffraction-limited image quality to IRDIS under nominal atmospheric conditions. It requires an $R<11$ star for the adaptive optics loop, but bright and compact extragalactic targets can also be used as guide sources as in the case of these observations. NGC 1068 was observed under rather good turbulence conditions (median seeing of about $1^{\prime \prime}$ ) on 10 and 11 December 2014. The adaptive optics correction quality was fair even though SAXO could not be used at full capacity owing to the faintness of the guide source. The airmass ranged from 1.1 to 1.26 during the observations. The achieved resolution on NGC 1068 (60 mas, i.e. about 4 pc) at $H(1.65 \mu \mathrm{m})$ and $K^{\prime}(2.2 \mu \mathrm{m})$ reveals new details over more than $600 \mathrm{pc}$ around the central engine. We built a data reduction pipeline to produce, for each band, maps with pixels of $0.01225^{\prime \prime}(0.9 \mathrm{pc})$ of the total intensity, the degree of linear polarization, and the linear 


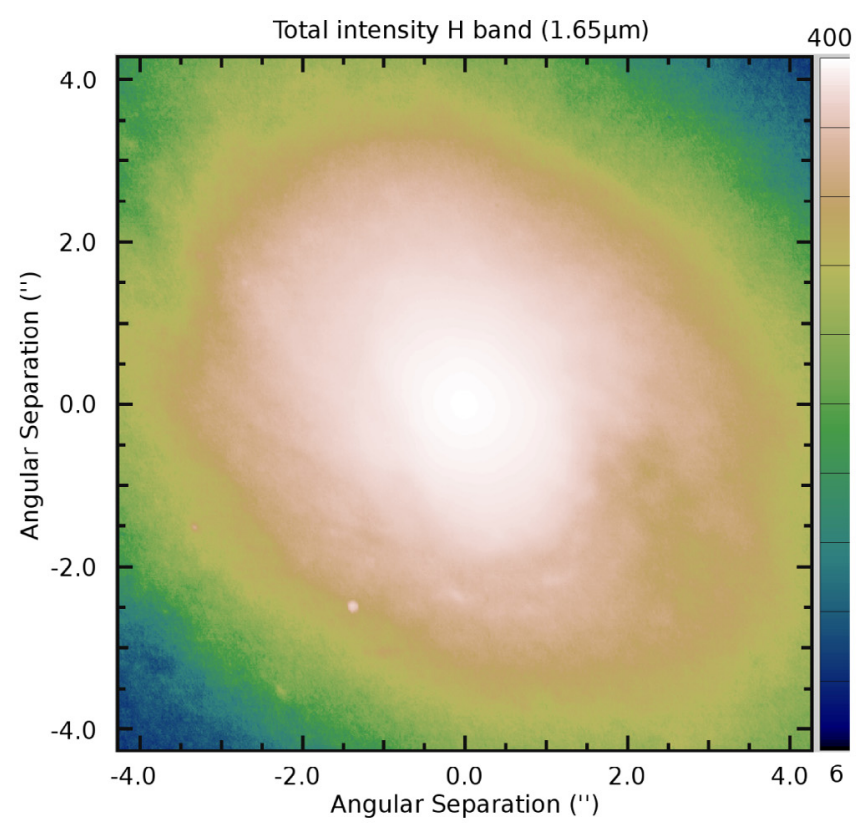

A

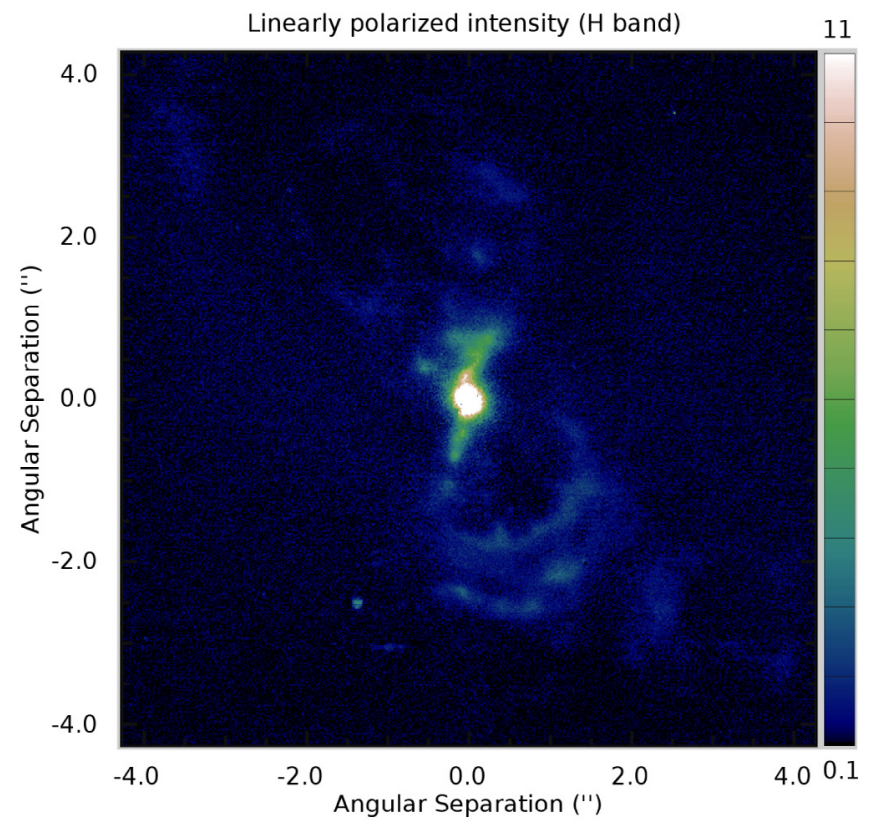

C

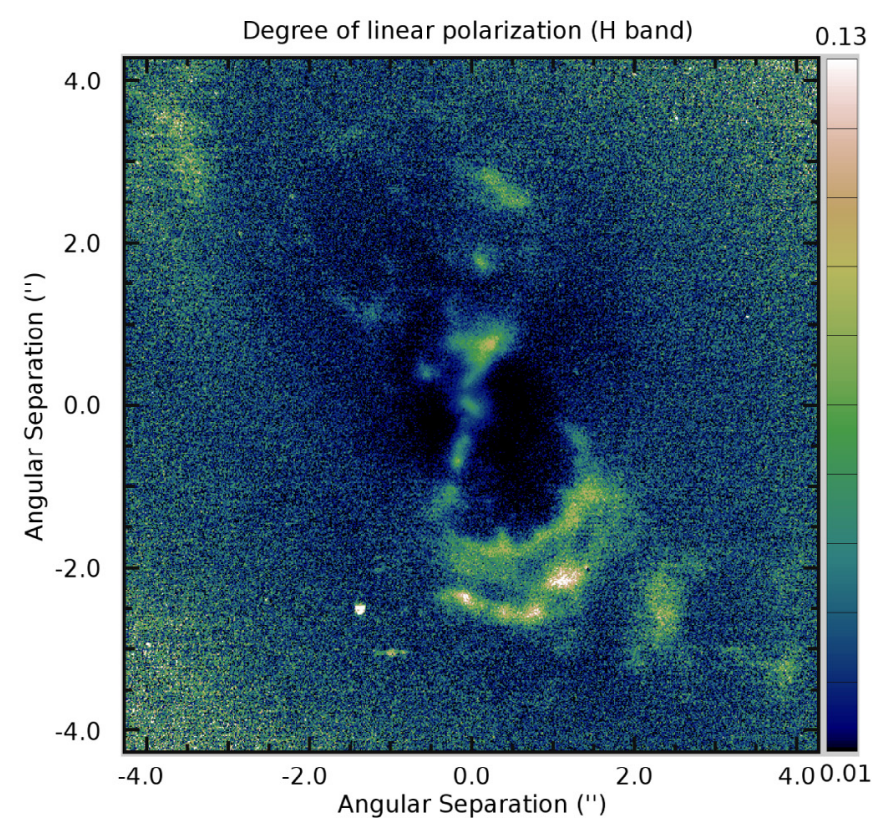

B

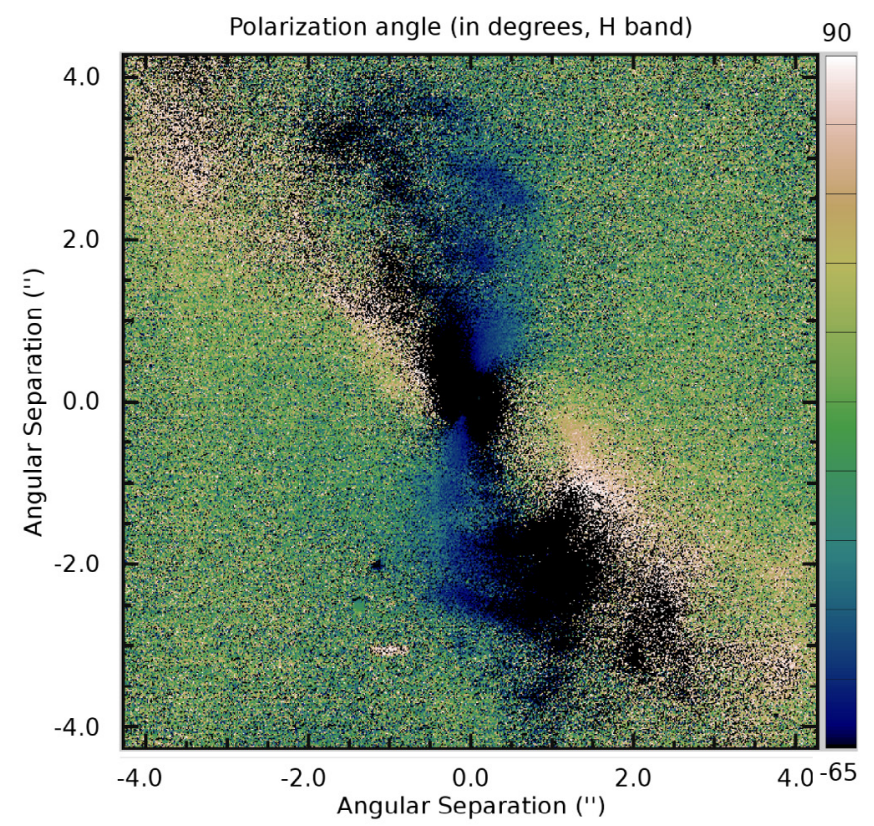

$\mathrm{D}$

Fig. 1. Output of our data reduction pipeline. North is up, east to the left. A) Total intensity image (color bar in arbitrary units); B) degree of linear polarization; C) polarized intensity (color bar in arbitrary units); D) polarization angle (in degrees). The total intensity image has been histogram-equalized between the 2 values in the color bar; i.e., each byte in the color map occurs with equal frequency between the 2 specified values.

polarization angle. This data reduction pipeline includes data pre-processing (detector cosmetics: flat-fielding and bad pixel correction, distortion correction: vertical spatial scale is multiplied by 1.006 , sky background subtraction and true north correction as measured on images of 47 Tuc), high accuracy shiftand-add (Gratadour et al. 2005a), and a dedicated polarimetric data reduction procedure. The latter is based on the double-ratio method, which is adequate for dual beam analyzers using halfwave plates (Tinbergen 1996). In such systems the polarization information is contained in the ratio of the two beams but mixed up with the system gain ratio for each pixel. To filter out the latter, Stokes parameters are obtained by computing the ratio of the intensity in one of the beams for two positions of the halfwave plate over the same ratio for the other beam. Four positions of the halfwave plate are thus required to retrieve the degree of polarization and the polarization angle. The total intensity is obtained by summing all the images from both beams. These maps are shown in Fig. 1 for the $H$ band with the addition of the polarized intensity image (Panel C), obtained from the product of the total intensity (Panel A) by the degree of polarization (Panel B). The total intensity image has been histogramequalized to help to show the nice spiral structure of the diffuse 
emission at low contrast, it is not representative of the real intensity image entirely dominated by the central source if displayed on a linear or logarithmic scale.

\section{Polarization in the ionization bicone}

At $H$ and at $K^{\prime}$, very similar polarized intensity images reveal a bright central source and a distinct bicone, whose axis is at position angle (PA) of about $30^{\circ}$. While the northern cone can be associated to the cloud complex of ionized gas that is extensively studied through optical ionization lines (Groves et al. 2004) and near-IR coronal lines (Barbosa et al. 2014), the southern cone appears as a perfectly symmetrical structure with respect to the central engine location. It shows distinct edges, which are highly polarized close to the base, as well as successive well-defined arcs at 125,170 , and 180 pc from the nucleus to the southwest. The orientation of the bicone with respect to the host galaxy explains that the southern cone is more conspicuous in the near-IR than in the visible (Das et al. 2006): the northern cone is above the disk, while the southern cone, below, is reddened. This is also consistent with the properties of the $21 \mathrm{~cm}$ absorption feature observed with the VLA (Gallimore et al. 1994). Highly polarized cone edges is consistent with a simple geometric assumption in which the edge-brightened regions have a scattering angle close to $90^{\circ}$ required for maximum polarization (Tadhunter et al. 1999). The southern arcs can be partly associated to patchy clouds detected in the optical with HST/FOC (Macchetto et al. 1994) and to features observed with coronagraphy in the near-IR (Gratadour et al. 2005b). The linearly polarized emission in the bicone is largely dominated by a centro-symmetric component, as shown by the map of polarization angle, and is thus interpreted as scattered emission from the central engine (Simpson et al. 2002). Kinematic models of a global outflow (conical or hourglass shaped) in the narrow line region (NLR), originating in a disk or torus wind, have been successfully compared to spectroscopic data in the optical (Das et al. 2006) and near-IR (Riffel et al. 2014) to explain the observed emission-line velocities in the whole bicone. In these models, the NLR clouds are accelerated to about $1000 \mathrm{~km} \mathrm{~s}^{-1}$ up to a distance of $80 \mathrm{pc}$ from the nucleus, keeping a constant velocity beyond (hourglass model) or decelerating to the galaxy's systemic (conical model), consistent with the location of the arc structure. The sharp and regular circular morphology of the near-IR polarized emission suggests a vast bow shock stemming from the interaction of this outflow with the galactic medium. It could explain the increased degree of polarization in these regions owing to the inescapable accumulation of matter, hence of dust, because of the velocity change at this location, resulting in an increase in Mie scattering efficiency. This interpretation is consistent with the lobe structures observed on radio images (see Wilson \& Ulvestad 1982 for instance) and infer the presence of a bow shock.

\section{Evidence for an extended nuclear torus}

We subtracted a purely centro-symmetric component from the map of polarization angle, assuming the central emitting component to be at the location of the near-IR peak intensity and with the proper reference angle, fit by minimizing the median residual angle over the bicone. Following this method, we find a PA for the bicone axis of $33^{\circ} \pm 2$. We are only interested in the absolute value of the difference between the directions of the polarization vectors and the centro-symmetric pattern (hence an angle between 0 and $90^{\circ}$ ). It is the quantity displayed in the lefthand panel of Fig. 2 for the $H$ band.
The result confirms that the polarized intensity in the bicone is completely dominated by scattered light from the central source. Most importantly, it reveals a compact elongated region in the inner arcsecond around the nucleus, showing a clear deviation from the centro-symmetric pattern with linear polarization aligned perpendicular to the bicone axis and extending over 55 pc perpendicular to this axis and about 20 pc parallel to it. The same result is obtained in both bands as shown by the magnified versions of the difference angle map around the nucleus for both the $H$ (left) and $K^{\prime}$ (right) bands pictured in the upper part of the right panel of Fig. 2. In addition to the lack of significant residual pattern in the peripheral zones on the difference image at each extremity of the elongated structure, we believe this feature is neither an instrumental artifact nor a shadow effect tracing a region where the polarization is cancelled totally due to multiple scattering in denser material closer to the nucleus. It should be noticed, though, that the bicone shape of this feature is an artefict inherent to the displayed parameter (difference of angle), Indeed for points on the ionization bicone axis, the apparent transverse component becomes parallel to the centro-symmetric vector so that the difference in angles vanishes along this axis. Most important, this subtraction process only enhances a pattern already observable on the polarization angle map without processing as shown by the image at the bottom of the righthand panel of Fig. 2.

In this image, the polarization vectors are displayed as bars overlaid on the degree of the linear polarization map. A transverse polarization angle component crossing the nucleus and extending over about $0.8^{\prime \prime}$ with the same PA can be clearly identified. Such a feature, with a central transverse polarization, has already been observed at lower angular resolution (Packham et al. 1997) and has been interpreted since as dichroic absorption of the central light radiation by aligned non-spherical dust grains. Additionally, it is believed that the transition between dichroic absorption and emission could explain the sudden flip of the polarization angle around $10 \mu \mathrm{m}$ (Packham et al. 2007) toward the nucleus. However, this interpretation appears incompatible with the observed extension of this feature appearing at high angular resolution because it would require a still undetected extended source of emission in the background to be attenuated by this structure.

This feature is similar to the polarimetric disk effect observed in young stellar objects. An original interpretation of this effect (Bastien \& Menard 1988) involves a thick disk or flat torus, on which the radiation from the central engine is scattered twice on average, before reaching the observer: once on the upper (or lower) surface of the disk and then near its external edge. Recently, refined radiative transfer modeling (Murakawa 2010) has supported this interpretation. In this model, grains can be spherical or not, and grain alignment is neither required nor expected to have a significant impact. These simulations show that an extended patch of linear polarization aligned in a direction perpendicular to the bicone axis can appear at its base for a thick disk (thickness parameter greater than 0.3) with rather small dust grains $(0.25 \mu \mathrm{m}$ diameter). The comparison of their Fig. 6 with our data, in the case of double scattering, is particularly convincing. Additionally, this model is able to reproduce the highly polarized bicone edges we observe.

In this simulation, the thickness of the disk, viewed almost edge-on, was taken to be $30 \%$ of the diameter, which is consistent with the thickness of $15 \mathrm{pc}$ measured at the outer edges of this feature on our data. For the effect to be efficient, the optical depth of the torus cannot be much larger than 1, otherwise multiple scattering would cancel the polarization: this condition is 
compatible with the range generally considered for NGC 1068. An optical depth on the line of sight of 1.25 in $K$ band is inferred, for instance, to cope with high angular resolution spectroscopic observations (Gratadour et al. 2003). We thus believe that this extended patch of linear polarization aligned in a direction perpendicular to the bicone axis is the first direct evidence of an extended torus at the core of NGC 1068.

The derived parameters (radius of $27 \mathrm{pc}$ and thickness of $15 \mathrm{pc}$ ) are consistent with recent results using ALMA data that complement a spectral energy distribution analysis (García-Burillo et al. 2014). Additionally, we derive a PA $118^{\circ}$ for the uniformly polarized structure that is consistent with the alignment of the two patches of hot molecular hydrogen observed in the near-IR (Müller Sánchez et al. 2009) and the orientation of the extended structure observed with VLBA at $5 \mathrm{GHz}$ (Gallimore et al. 2004). Moreover, the map of degree of polarization seems to suggest that the obscuring complex extends way beyond the elongated feature we detect around the core, with two lobes of extremely low polarization levels on each side of the nucleus showing efficient screening from the central radiation.

While the polarization level in this extended patch is rather low, which agrees with the polarized disk model, one thing the latter cannot reproduce is the ridge of higher linear polarization observed at the location of the nucleus with a PA of $56^{\circ}$ (center of bottom right panel in Fig. 2). We propose that this ridge be interpreted as the location at which the linearly polarized emission due to dichroic absorption of the central light originates. While this effect was not included in the other simulations we mentioned, the measured degree of polarization (5-7\%) is consistent with such an interpretation. The size of the minor axis of this feature is consistent with the size of the dust sublimation cavity, believed to be the main contributor to the near-IR emission from the core, as it would appear convolved by the instrumental PSF. However, its extension (comparable to the thickness of the previously inferred torus) and exact orientation are questionable.

A deeper analysis of our data, including a close comparison of $H$ and $K^{\prime}$ bands and numerical simulations, including dichroic absorption, are required for a better understanding of the nature of this ridge.
Acknowledgements. This paper is based on observations at the Very Large Telescope (VLT) of the European Observatory (ESO) in Chile. We thank Julien Girard and Dimitri Mawet for carrying out the observations and Maud Langlois for helpful discussions of how to calibrate polarimetric observations with SPHERE, along with Chris Packham and Enrique Lopez-Rodriguez for very useful and lively discussions regarding data interpretation.

\section{References}

Antonucci, R. 1993, ARA\&A, 31, 473

Antonucci, R. R. J., \& Miller, J. S. 1985, ApJ, 297, 621

Barbosa, F. K. B., Storchi-Bergmann, T., McGregor, P., Vale, T. B., \& Rogemar Riffel, A. 2014, MNRAS, 445, 2353

Bastien, P., \& Menard, F. 1988, ApJ, 326, 334

Beuzit, J.-L., Feldt, M., Dohlen, K., et al. 2008, in SPIE Conf. Ser., 7014, 18

Das, V., Crenshaw, D. M., Kraemer, S. B., \& Deo, R. P. 2006, AJ, 132, 620

Exposito, J., Gratadour, D., Clénet, Y., \& Rouan, D. 2011, A\&A, 533, A63

Gallimore, J. F., Baum, S. A., O’Dea, C. P., Brinks, E., \& Pedlar, A. 1994, ApJ, 422, L13

Gallimore, J. F., Baum, S. A., \& O’Dea, C. P. 2004, ApJ, 613, 794

García-Burillo, S., Combes, F., Usero, A., et al. 2014, A\&A, 567, A125

Gratadour, D., Clénet, Y., Rouan, D., Lai, O., \& Forveille, T. 2003, A\&A, 411, 335

Gratadour, D., Mugnier, L. M., \& Rouan, D. 2005a, A\&A, 443, 357

Gratadour, D., Rouan, D., Boccaletti, A., Riaud, P., \& Clénet, Y. 2005b, A\&A, 429,433

Gratadour, D., Rouan, D., Mugnier, L. M., et al. 2006, A\&A, 446, 813

Groves, B. A., Cecil, G., Ferruit, P., \& Dopita, M. A. 2004, ApJ, 611, 786

Langlois, M., Dohlen, K., Vigan, A., et al. 2014, in SPIE Conf. Ser., 9147, 1

Macchetto, F., Capetti, A., Sparks, W. B., Axon, D. J., \& Boksenberg, A. 1994, ApJ, 435, L15

Müller Sánchez, F., Davies, R. I., Genzel, R., et al. 2009, ApJ, 691, 749

Murakawa, K. 2010, A\&A, 518, A63

Nenkova, M., Ivezić, Ž., \& Elitzur, M. 2002, ApJ, 570, L9

Packham, C., Young, S., Hough, J. H., Axon, D. J., \& Bailey, J. A. 1997, MNRAS, 288, 375

Packham, C., Young, S., Fisher, S., et al. 2007, ApJ, 661, L29

Raban, D., Jaffe, W., Röttgering, H., Meisenheimer, K., \& Tristram, K. R. W. 2009, MNRAS, 394, 1325

Riffel, R. A., Vale, T. B., Storchi-Bergmann, T., \& McGregor, P. J. 2014, MNRAS, 442, 656

Rouan, D., Lacombe, F., Gendron, E., et al. 2004, A\&A, 417, L1

Simpson, J. P., Colgan, S. W. J., Erickson, E. F., et al. 2002, ApJ, 574, 95

Tadhunter, C. N., Packham, C., Axon, D. J., et al. 1999, ApJ, 512, L91

Tinbergen, J. 1996, Astronomical Polarimetry (Cambridge, UK: Cambridge University Press)

Wilson, A. S., \& Ulvestad, J. S. 1982, ApJ, 263, 576

Page 5 is available in the electronic edition of the journal at http://www . aanda.org 

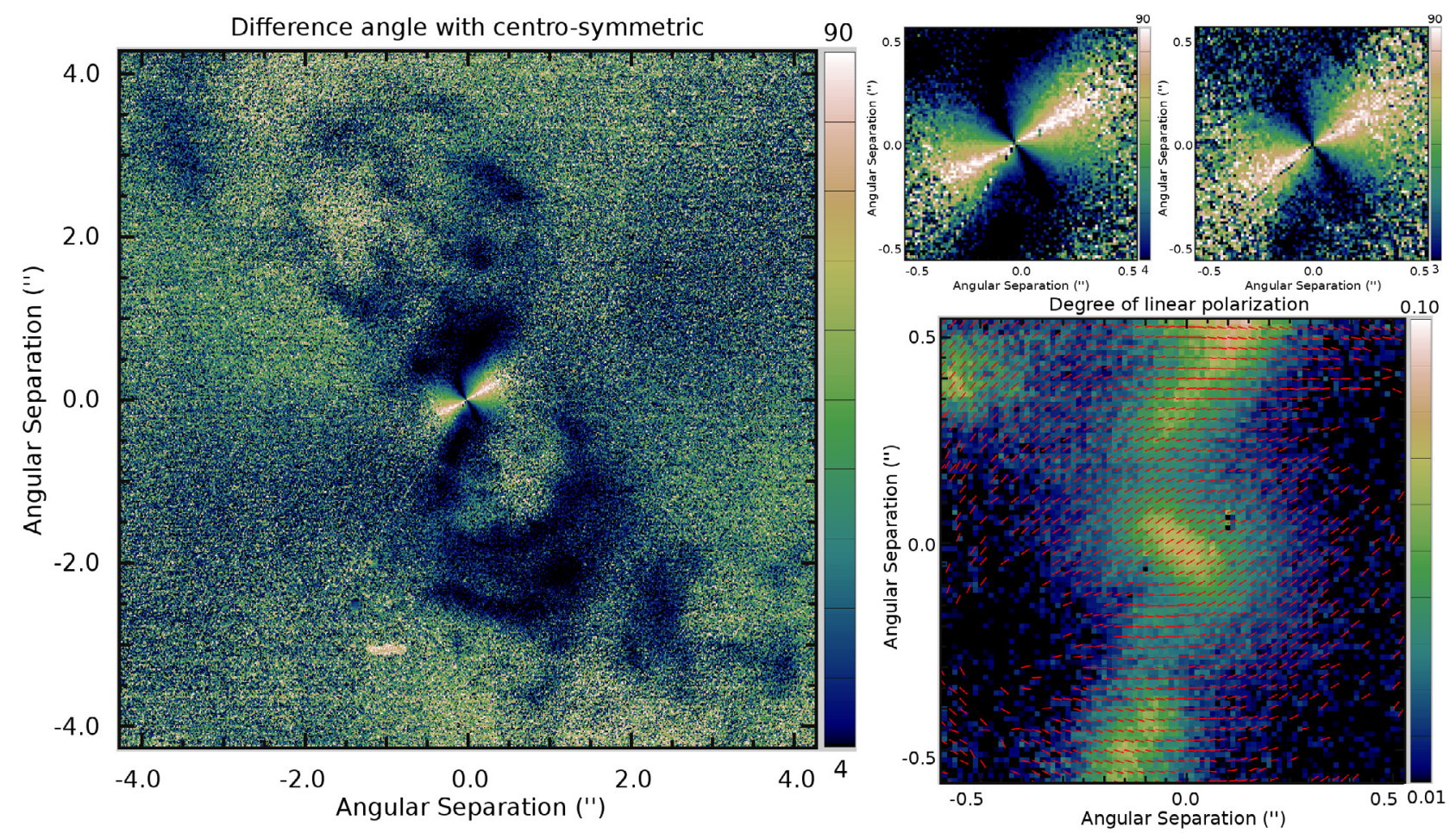

Fig. 2. Left panel: result of the difference between the polarization angle map and a purely centro-symmetric pattern. Right panel: the top left image is a magnified version of the left panel, around the central source and top right is the result of the same processing on the $K^{\prime}$ band angle map. The bottom right image is a magnified version of the degree of linear polarization found in Fig. 1, on which we overlaid the direction of the polarization vectors, showing the clear patch of aligned vectors in this region in the data before the subtraction of the centro-symmetric pattern. 